\title{
Abolishing Trp53-dependent apoptosis does not benefit spinal muscular atrophy model mice
}

\author{
Ming Shiun Tsai ${ }^{1,2}$, Yung Tsung Chiu ${ }^{3}$, Sue Hong Wang ${ }^{4}$, Hsiu Mei Hsieh-Li ${ }^{5}$ and Hung $\mathrm{Li}^{*, 1}$
}

${ }^{1}$ Institute of Molecular Biology, Academia Sinica, Taipei, Taiwan; ${ }^{2}$ Department of Bioindustry Technology, Da-Yeh University, Da-Tsuen, Chang-Hua, Taiwan; ${ }^{3}$ Laboratory of Comparative Pathology, department of Education and Research, Taichung Veterans General Hospital, Taichung, Taiwan; ${ }^{4}$ Department of Biomedical Science, Chung Shan Medical University, Taichung, Taiwan; ${ }^{5}$ Department of Life Science, National Taiwan Normal University, Taipei, Taiwan

Spinal muscular atrophy (SMA) is the most common genetic motoneuron degenerative disorder, but the mechanism(s) of motoneuron death is unclear. Previously, a direct interaction between tumor-suppressive TP53 protein and the SMA determinant gene product, survival motor neuron protein, was identified and therefore it has been suggested that a mechanism of TP53-dependent apoptosis plays an important role in motoneuron degeneration in SMA. We used our SMA model mice, generated by a combination of knockout and transgenic techniques, to decipher the role of TP53 protein in the motoneuron degeneration in SMA. We detected a significant increase of Trp53 expression in the spinal cord of SMA-like mice compared to their normal littermates. After crossing SMA-like mice with Trp53 knockout mice, the progeny Trp53-deficient SMA-like mice did not show milder disease severity or longer lifespan compared to SMA littermates with wild-type Trp53 genes. Our studies provide in vivo evidence indicating that Trp53dependent apoptosis does not play a crucial role in motoneuron degeneration in SMA-like mice. European Journal of Human Genetics (2006) 14, 372-375. doi:10.1038/sj.ejhg.5201556; published online 4 January 2006

Keywords: Trp53-dependent apoptosis; spinal muscular atrophy; motoneuron degeneration; SMA model mice

\section{Introduction}

Spinal muscular atrophy (SMA), an autosomal recessive motoneuron degenerative disorder, is caused by degeneration of the spinal motoneurons leading to muscular paralysis and atrophy. ${ }^{1}$ SMA patients are clinically categorized, on the basis of age of disease onset and clinical course, into three types: SMA 1 (the most severe, Werdnig-Hoffmann disease, MIM253300), SMA 2 (the intermediate, MIM253550), and SMA 3 (the mildest, Kugelberg-Welander disease, MIM253400). ${ }^{2}$ Mutations of the SMA determinant gene, survival motor neuron (SMN1) gene, are found in about $96 \%$ of human SMA patients. ${ }^{3}$ Although the relationship between SMA and the SMN1 gene product has been known

*Correspondence: $\mathrm{Dr} \mathrm{H} \mathrm{Li}$, Institute of Molecular Biology, Academia Sinica, Nankang, Taipei 11529, Taiwan. Tel: +886 2 27880460;

Fax: + 8862 27826085; E-mail: hungli@gate.sinica.edu.tw

Received 8 June 2005; revised 11 November 2005; accepted 15 November 2005; published online 4 January 2006 for years, the neurodegeneration pathways responsible for SMA motoneuron death remain unclear. ${ }^{1}$ Previously, we created a SMA mouse model by a combination of knockout and transgenic techniques. ${ }^{4}$ The offspring SMA-like mice with homologous mouse Smn gene knockouts and hemizygous human SMN2 transgenes (genotype: Smn ${ }^{-/-}$ $S M N 2^{+/-}$), genotypically and phenotypically mimic SMA patients, and they can be categorized into three clinical types in the same way as human patients according to the phenotypes and survival times. ${ }^{4}$ Therefore, these SMA-like mice are suitable for use in studying the mechanism of SMA motoneuron degeneration.

Tumor-suppressive TP53 protein, which can induce cell cycle arrest and apoptosis, performs a critical role in the regulation of neuronal differentiation and apoptosis. ${ }^{5,6}$ TP53 can induce neuronal death through a Bax-dependent pathway by directly transactivating proapoptotic Bax expression. ${ }^{7,8}$ Recently, TP53 was reported to directly interact with SMN protein, ${ }^{9}$ and therefore TP53-dependent 
apoptotic pathways were suggested to play a crucial role in the motoneuron loss in SMA disease. ${ }^{9,10}$ To test this hypothesis, in the present study we used our SMA model mice to cross the Trp53 knockout mice. ${ }^{11}$ The offspring Trp53-deficent SMA mice did not show milder disease severity or longer lifespan than their SMA littermates with wild-type Trp53 genes, but some of them showed different phenotypes and neurodegeneration in the brain stems. This constitutes in vivo evidence against the hypothesis that TP53-dependent apoptotic pathways play a crucial role in motoneuron loss in SMA disease.

\section{Materials and methods \\ Mice}

Transgenic mice containing two copies of human SMN2 genes ${ }^{4}$ were crossed with heterozygous mouse Smn knockout mice. The offspring heterozygous Smn knockout mice with or without SMN2 transgenes were intercrossed to generate SMA-like mice (genotypes: $S m n^{-/} S M N 2^{+/-}$). We generated the double heterozygous knockout mice (Smn and Trp53) with or without SMN2 transgenes by crossing homozygous Trp53 knockout mice (P53N4-M, Taconic Farm) with the $S m n^{+/-} S M N 2^{+/-}$mice. We obtained SMAlike mice with different Trp53 genotypes by intercrossing double heterozygous mice with and without SMN2 transgenes. Mice were supplied with sterile water ad libitum and rodent pellets, and were under the care of the animal facility of the Institute of Molecular Biology, Academia Sinica. All procedures were approved by the Academia Sinica Animal Care and Use Committee, Master Protocol \# RMiRbIMBLH2001132.

We analyzed the genotypes of Smn knockout, SMN2 transgenic, and Trp53 knockout mice by polymerase chain reaction (PCR) analysis. Mouse tail genomic DNAs were prepared as previously described. ${ }^{4}$ The three specific PCR primers (S1, S2, and H1) were used for detection of the wild-type and knockout Smn alleles, and the primer pair (3F and 3B) were used to identify the human SMN2 transgenes. ${ }^{4}$ The three specific primers (primer $4 \mathrm{~b}$, primer 6 , and primer in the polII promoter region) were used to identify the wild-type and knockout Trp53 alleles. ${ }^{11}$ For statistical analyses of life spans of mice, differences were compared between two groups by the Student's $t$-test. The results were considered to be significant when $P<0.05$.

\section{Western blotting, histopathological and immuno- staining analyses}

We isolated spinal cords from three different clinical types of SMA-like mice and their normal littermates that had been freshly killed. Total proteins were extracted from the spinal cords using extraction buffer containing $0.4 \%$ Nonidet $P-40,0.1 \%$ sodium dodecylsulfate (SDS), and proteinase inhibitors (Complete ${ }^{\mathrm{TM}}$ proteinase inhibitor Cocktail, Roche Biomedical). Equal amounts of extracted proteins were loaded and proteins were separated by $12 \%$
SDS-polyacrylamide gel electrophoresis. ${ }^{12}$ After transferring to polyvinyl difluoride membranes (Millipore), we blocked the membranes in TBST buffer containing nonskimmed milk. All antibodies used were purchased from Santa Cruz Biotechnology. Adequate dilutions as per the manufacturer's suggestion for anti- $\beta$-actin (I-19), anti-p53 (FL-393), and anti-SMN (N-19) antibodies were reacted. Secondary antibodies conjugated with horseradish peroxidase were then used. We detected the reactions by chemiluminescence (Chemiluminescence Reagent Plus, PerkinElmer Life Sciences) and signals were quantified by computer densitometer (Amersham Pharmacia Biotech). The expression levels of Trp53 were compared with $\beta$-actin. Increases of Trp53 expression in different clinical types of SMA-like mice were obtained and compared with values for their normal littermates. These increases were indicated as mean values \pm SD from three independent experiments.

After killing mice, brains and spinal cords were removed and fixed in 10\% neutral-buffered formalin (Fisher Scientific) and then embedded in paraffin. Thick transverse sections, $6 \mu \mathrm{m}$, were stained with hematoxylin and eosin ${ }^{4}$ and observed under light microscope (ZEISS, Axioskop2). The images were captured electronically by CoolSNAP-pro, Media Cybernetic, and were analyzed using Image Pro ${ }^{\circledR}$ Plus version 4.1 .

\section{Results and discussion}

In order to investigate the relationship between Trp53 protein and SMA motoneuron death, we analyzed the expression levels of Trp53 protein within spinal cords of type 1 SMA-like mice and their normal littermates by Western blotting. Dramatically lower SMN levels (about one-tenth) were detected in the spinal cord extracts from type 1 SMA-like mice in comparison with the spinal cord extracts of their normal age-matched littermates, and levels of $\beta$-actin were detected as an internal control (Figure 1a). We found a significant increase in Trp53 expression $(2.78 \pm 0.43$ fold) in type 1 SMA-like mice in comparison with their normal littermates (Figure 1a). Furthermore, a significant increase in Trp53 expression $(1.96 \pm 0.36$ fold) was also found in type 2 SMA-like mice but not in type 3 SMA-like mice $(1.11 \pm 0.41$ fold $)$ in comparison with their normal littermates (Figure 1b). Since the more severe SMA-like mice showed the more significant increase in Trp53 expression in their spinal cords, the increase in Trp53 expressions correlated with the disease severities of SMA in SMA-like mice.

To determine the in vivo role of Trp53 protein in SMA motoneuron degeneration, we crossed knockout-transgenic mice (genotype: $S m n^{+/-} S M N 2^{+/-}$) with Trp53 knockout mice (genotype: $\left.\operatorname{Tr} p 53^{-/-}\right)^{11}$ to generate double heterozygous knockout mice (genotype: $\mathrm{Smn}^{+/-} \operatorname{Trp53}{ }^{+/-}$) with or without SMN2 transgenes. The Trp53-/- mice were deficient in Trp53 protein expression, but SMN protein expression was not affected (Figure 1c). This result indicated 


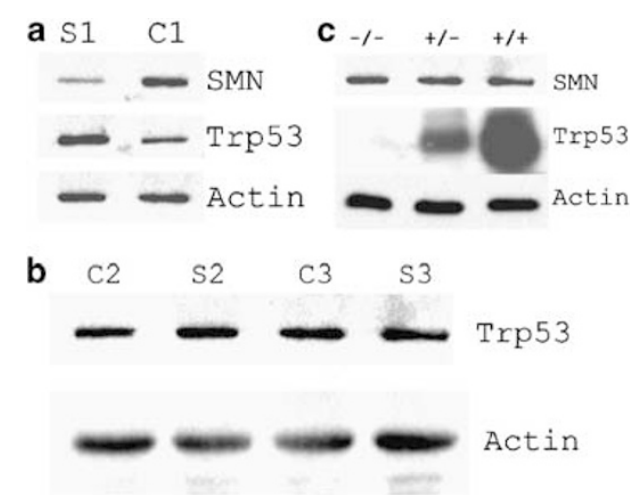

Figure 1 Analysis of protein expression levels in the mouse spinal cords. (a) Expression levels of $\operatorname{Trp} 53, \mathrm{SMN}$, and $\beta$-actin were detected in the spinal cords of type 1 SMA-like mice (S1) and age-matched normal littermates (C1). (b) Expression levels of Trp53 and $\beta$-actin were detected in the spinal cords of type 2 (S2) and type 3 (S3) SMAlike mice and their age-matched normal littermates (C2 and C3). (c) Expression levels of Trp53, SMN, and $\beta$-actin were detected in the spinal cords of homozygous $(-/-)$ and heterzygous $(+/-)$ Trp53 knockout mice and their wild-type littermate $(+/+)$.

that Trp53 did not regulate the expression of SMN protein. We then crossed $S m n^{+/-} \operatorname{Tr} 553^{+/-} S M N 2^{-/-}$mice with Smn ${ }^{+/-}$Trp53 $3^{+1-}$ SMN2 $2^{+1-}$ mice to generate SMA-like mice (genotype: $\mathrm{Smn}^{-1-} \mathrm{SMN2} \mathrm{2}^{+/-}$) with different Trp53 genotypes. After breeding and genotyping more than a thousand offspring mice, we obtained the survival data of SMA-like mice with $\operatorname{Tr} 553^{-/-}$and $\operatorname{Tr} 553^{+/+}$genotypes bred by the same parents. Totals of 11 pups with type 1 , six pups with type 2 , and six pups with type 3 SMA were obtained with Trp53 $3^{-/}$genotype; in addition, 14 pups with type 1,16 pups with type 2 , and four pups with type 3 SMA were obtained with $\operatorname{Tr} p 53^{+/+}$genotype. Although the theoretical probabilities of breeding SMA-like mice with $\operatorname{Tr} 553^{-/-}$or $\operatorname{Trp53} 3^{+1+}$ genotypes are the same, the total number of Trp53 $3^{-1-}$ SMA mice $(n=23)$ obtained was much lower than $\operatorname{Trp} 53^{+1+}$ SMA mice $(n=34)$. This might be because of embryonic or neonatal lethality caused by Trp53 deficiency. Furthermore, we obtained over 20 dead neonatal mice containing Trp53 knockout but not Smn knockout genotypes (data not shown); we can conclude these mice died because of Trp53 deficiency but not SMA. The average lifespan of type 1 Trp53 $3^{-1-}$ SMA mice was $6.73 \pm 1.45$ days, which was significantly shorter than type $1 \operatorname{Trp}^{2} 3^{+/+}$SMA mice with $8.57 \pm 1.03$ days $(P<0.03)$. The decrease in average lifespan of type 1 Trp53 $3^{-/}$SMA mice might be the result of the neonatal lethality caused by Trp53 deficiency. Furthermore, the lifespans of type 3 Trp53 ${ }^{-1-}$ SMA mice (from 2 to 5 months) were also shorter than lifespans of type $3 \operatorname{Trp5} 3^{+/+}$SMA mice (from 6 to 15 months). Type 3 Trp53 $3^{-1-}$ SMA mice showed various tumor formations, as previously presented. ${ }^{13}$ Since Trp53 deficiency may result in spontaneous tumor formation, ${ }^{11,13}$ the shorter lifespan of type 3 Trp53 $3^{-1-}$ SMA mice can probably be attributed to tumor formation. However, the average lifespan of type 2
$\operatorname{Trp53} 3^{-/-}$SMA mice $(17.33 \pm 3.63$ days $)$ was significantly longer than lifespan of type $2 \operatorname{Trp} 53^{+1+}$ SMA mice $(13.38 \pm 2.21$ days $)(P<0.04)$. Therefore, the survival curves of $\operatorname{Tr} p 53^{-1-}$ SMA and Trp53 $3^{+1+}$ SMA mice were inter-crossed (Figure 2). The probabilities of survival for mice were higher before postnatal day 10 and lower after postnatal day 11 in the $\operatorname{Tr} p 53^{+/+}$SMA than in the $\operatorname{Tr} p 53^{-/-}$SMA mice groups. From these results, we can not conclude that Trp53 deficiency prolongs the lifespan of SMA-like mice.

Type $2 \operatorname{Trp} 53^{-1-}$ SMA mice that showed severe atrophy of the tail and lower limbs, as well as bleeding from the lower abdomen were obtained (Figure 3a). However, these phenotypes were not found in type $2 \operatorname{Tr} 553^{+/+}$SMA mice. Furthermore, type 3 Trp53 ${ }^{-1-}$ SMA mice showed neuronal degeneration in their brain stems that were not detected in their type 3 Trp $53^{+/+}$SMA littermates (Figures 3b-e). These results suggest the possibility that Trp53 deficiency in SMA-like mice changed the pathophysiology and symptoms of SMA disease. The mechanism of this change needs to be further analyzed.

We previously found in vivo evidence that showed that Bax-dependent apoptosis played a crucial role in motoneuron degeneration in SMA (Tsai et al, unpublished data). Here, we detected an increase in Trp53 expression in spinal cords of type 1 and type 2 SMA-like mice compared to normal littermates (Figure 1a and b). An increase in Trp53 expression was also detected in the spinal motoneurons of amyotrophic lateral sclerosis 1 (ALS1, MIM105400) model mice. ${ }^{14}$ Furthermore, TP53 can directly interact with SMN protein; a decrease in SMN protein expression in SMA patients inhibited TP53SMN complex formation, thus promoting the translocation of TP53 into nucleolus. ${ }^{9}$ Therefore, a possible mechanism for motoneuron loss by TP53-dependent apoptosis has been suggested for both ALS and SMA

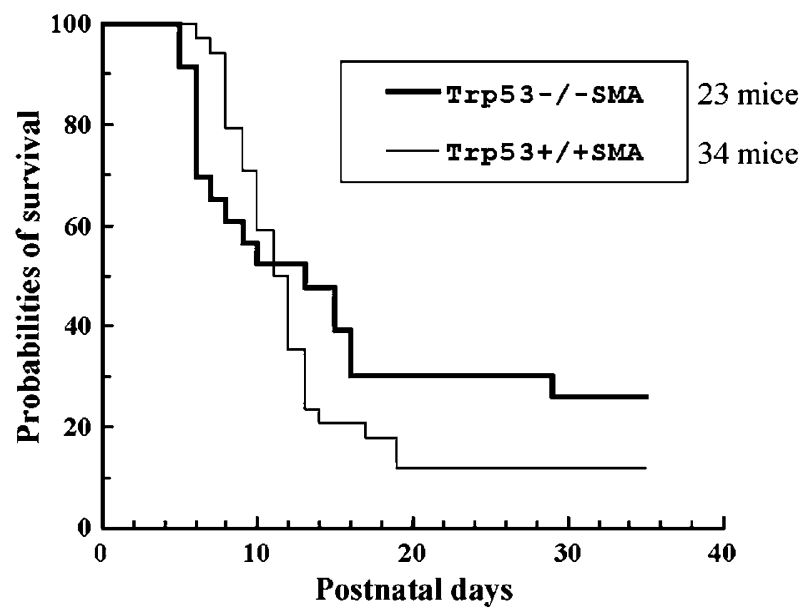

Figure 2 Survival curves of offspring SMA-like mice with different $\operatorname{Trp53}$ genotypes. Survival curves of SMA littermates with $\operatorname{Trp5} 53^{+1+}$ and $\operatorname{Trp}_{53^{-1}}$ genotypes were presented as Kaplan-Meier plots. Numbers of mice obtained were as indicated. 

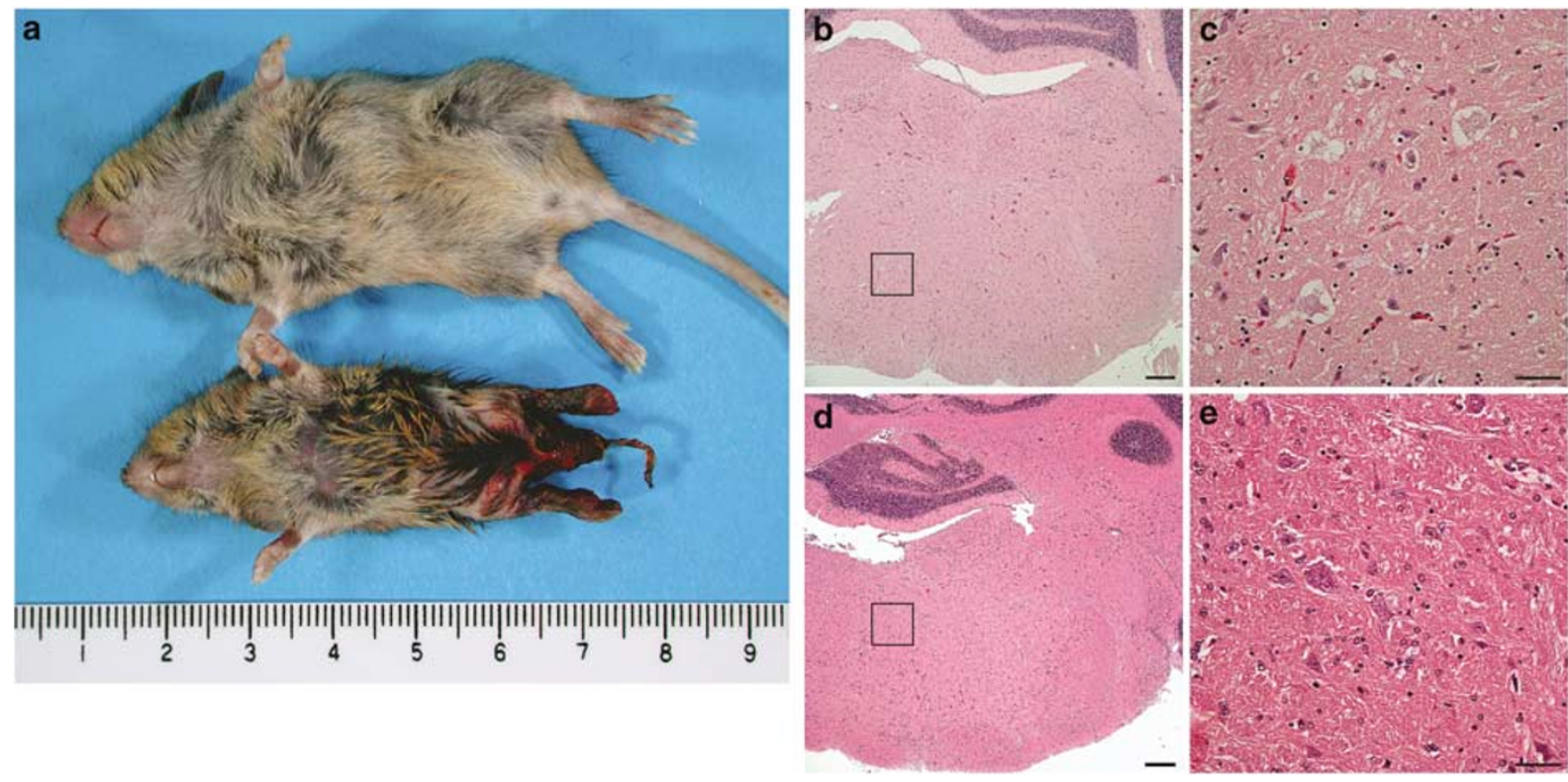

Figure 3 Phenotypes and histopathological analysis of Trp53-deficient SMA-like mice and their wild-type or SMA littermates. (a) Images of a type 2 Trp53-deficient SMA-like mouse and its normal littermate at 22 postnatal days. Histopathological findings of cross-sections from the brain stems of a 3 month-old type $3 \mathrm{Trp5} 3^{-1-}$ SMA mouse (b and $\mathbf{c}$ ) and its type $3 \mathrm{Trp5} 3^{+/+}$SMA littermate (d and e). Panel $\mathbf{c}$ and panel e are enlarged figures of the boxed regions from panel $b$ and panel $d$, respectively. Vesicles to vacuolar degenerations of motoneurons in the brain stem can be seen (c). Scale bars: $250 \mu \mathrm{m}$ (b and d); $40 \mu \mathrm{m}$ (c and e).

diseases. ${ }^{9,14}$ However, our results, obtained by crossing SMA-like mice with Trp53 knockout mice, do not support this hypothesis. Although type 2 Trp5 $53^{-1-}$ SMA mice showed a longer lifespan than their type 2 SMA littermates with wild-type $\operatorname{Tr} p 53$ genes, Trp53 deficiency was detrimental to the survival of both type 1 and type 3 SMA-like mice. In addition, Trp53 deficiency might cause alteration of the phenotypes and pathophysiology of type 2 and type 3 SMA-like mice (Figure 3). If Trp53-dependent apoptosis played a crucial role in motoneuron degeneration of SMAlike mice, Trp53 deficiency would protect the spinal motoneurons from death and result in Trp53-deficient SMA-like mice living longer. However, we can not obtain this conclusion from this study. Our results do not support the hypothesis that inhibition of Trp53-dependent apoptosis shows beneficial effect on a SMA model mice, although this does not rule out a different role for Trp53 in SMA patients.

\section{Acknowledgements}

We thank Dr K Deen for his critical reading of manuscript and Ms. Ping-Jui Chen for her great help in the preparation of mouse tail DNAs. This work was supported in part by the National Health Research Institute (Grant NHRI-EX92-9029SP).

\section{References}

1 Jablonka S, Sendtner M: Molecular and cellular basis of spinal muscular atrophy. Amyotroph Lateral Scler Other Mot Neuron Disord 2003; 4: 144-149.
2 Munsat TL, Davies KE: International SMA consortium meeting. Neuromuscul Disord 1992; 2: 423-428.

3 Wirth B: An update of the mutation spectrum of the survival motor neuron gene (SMN1) in autosomal recessive spinal muscular atrophy (SMA). Hum Mutat 2000; 15: 228-237.

4 Hsieh-Li HM, Chang JG, Jong YJ et al: A mouse model for spinal muscular atrophy. Nat Genet 2000; 24: 66-70.

5 Eizenberg O, Faber-Elman A, Gottlieb E, Oren M, Rotter V, Schwartz M: p53 plays a regulatory role in differentiation and apoptosis of central nervous system-associated cells. Mol Cell Biol 1996; 16: 5178-5185.

6 Vousden KH: p53: death star. Cell 2000; 103: 691-694.

7 Xiang H, Kinoshita Y, Knudson CM, Korsmeyer SJ, Schwartzkroin PA, Morrison RS: Bax involvement in p53-mediated neuronal cell death. J Neurosci 1998; 18: 1363-1373.

8 Miyashita T, Reed JC: Tumor suppressor p53 is a direct transcriptional activator of the human bax gene. Cell 1995; 80: 293-299.

9 Young PJ, Day PM, Zhou J, Androphy EJ, Morris GE, Lorson CL: A direct interaction between the survival motor neuron protein and p53 and its relationship to spinal muscular atrophy. J Biol Chem 2002; 277: 2852-2859.

10 Gostissa M, Hofmann TG, Will H, Del Sal G: Regulation of p53 functions: let's meet at the nuclear bodies. Curr Opin Cell Biol 2003; 15: 351-357.

11 Donehower LA, Harvey M, Slagle BL et al: Mice deficient for p53 are developmentally normal but susceptible to spontaneous tumours. Nature 1992; 356: 215-221.

12 Laemmli UK: Cleavage of structural proteins during the assembly of the head of bacteriophage T4. Nature 1970; 227: 680-685.

13 Attardi LD, Jacks T: The role of p53 in tumour suppression: lessons from mouse models. Cell Mol Life Sci 1999; 55: 48-63.

14 Gonzalez de Aguilar JL, Gordon JW, Rene F et al: Alteration of the $\mathrm{Bcl}-\mathrm{x} / \mathrm{Bax}$ ratio in a transgenic mouse model of amyotrophic lateral sclerosis: evidence for the implication of the p53 signaling pathway. Neurobiol Dis 2000; 7: 406-415. 